\title{
Tecnologías de internet de las cosas en la obtención de información
}

\section{Internet Technologies things in obtaining information}

\section{Tecnologias de internet das coisas na obtenção de informações}

\author{
Marely del R. Cruz-Felipe ${ }^{\mathrm{I}}$ \\ marely.cruz@fci.edu.ec \\ Jenmer M. Pinargote-Ortega ${ }^{\mathrm{II}}$ \\ maricela.pinargote@fci.edu.ec \\ Gabriel P. Demera-Ureta ${ }^{\text {III }}$ \\ gabriel.demera@fci.edu.ec \\ Dannyll M. Zambrano-Zambrano ${ }^{\text {IV }}$ \\ michel.zambrano@fci.edu.ec
}

Recibido: 15 de octubre de 2017 * Corregido: 19 de noviembre de 2017 * Aceptado: 21 de febrero de 2018

I Doctor en Ciencias Técnicas, Ingeniero en Equipos y Componentes Electrónicos, Docente de la Universidad Técnica de Manabí, Portoviejo, Ecuador.

II Magister en Pedagogía, Especialista en Pedagogía, Diploma Superior en Pedagogía, Ingeniera en Sistemas Informáticos, Docente de la Universidad Técnica de Manabí, Portoviejo, Ecuador.

III Magister en Docencia e Investigación Educativa, Ingeniero en Sistemas Computacionales, Docente de la Universidad Técnica de Manabí, Portoviejo, Ecuador.

IV Magister en Redes de Comunicaciones, Ingeniero en Electrónica y Telecomunicaciones, Docente de la Universidad Técnica de Manabí, Portoviejo, Ecuador. 
Tecnologías de internet de las cosas en la obtención de información

\title{
Resumen
}

La evolución de Internet ha marcado nuevas formas de acceder a la información. Una de las más recientes es el empleo de esta red para acceder a todo tipo de objeto que pueda ser censado lo que llamamos Internet de las cosas. Sin dudas esta nueva forma brinda diversas posibilidades en todos los sectores de la humanidad. La investigación utilizó como método teórico, el analítico-sintético para realizar un análisis de las diferentes posibilidades que brinda Internet de las cosas y las tecnologías necesarias para el empleo de la misma resaltando los diferentes tipos de información que se pueden obtener con cada una y la aplicación que proporcionan. En la que se concluye, que en los próximos años el internet de las cosas tendrá una gran participación en el desarrollo de las actividades del ser humano en función de la evolución de herramientas que permitan la interconexión a través de internet.

Palabras clave: internet de las cosas; objetos; RFID; IdC.

\begin{abstract}
The evolution of the Internet has marked new ways to access information. One of the most recent is the use of this network to access all kinds of objects that can be called what we call the Internet of things. Without doubts this new form offers diverse possibilities in all the sectors of the humanity. The research used as a theoretical method, the analytical-synthetic to perform an analysis of the different possibilities offered by the Internet of things and the technologies necessary for the use of it, highlighting the different types of information that can be obtained with each and the application they provide. In which it is concluded, that in the coming years the Internet of Things will have a great participation in the development of human activities in function of the evolution of tools that allow interconnection through the Internet.
\end{abstract}

Keywords: internet of things; objects; RFID; IdC.

\section{Resumo}

A evolução da Internet marcou novas formas de acessar informações. Um dos mais recentes é o uso dessa rede para acessar todos os tipos de objetos que podem ser chamados de Internet das coisas. Sem dúvidas esta nova forma oferece diversas possibilidades em todos os setores da humanidade. A 
pesquisa utilizou como método teórico, o analítico-sintético para realizar uma análise das diferentes possibilidades oferecidas pela Internet das coisas e as tecnologias necessárias para o uso do mesmo, destacando os diferentes tipos de informações que podem ser obtidas com cada aplicação que eles fornecem. Em que se conclui, que nos próximos anos a Internet das Coisas terá uma grande participação no desenvolvimento de atividades humanas em função da evolução de ferramentas que permitam a interconexão através da Internet.

Palavras chave: internet das coisas; objetos; RFID; IdC.

\section{Introducción}

Desde el surgimiento de Internet, este ha revolucionado la forma de acceder a la información y por consiguiente sus beneficios han sido aplicados rápidamente en todas las esferas de la sociedad, hace 20 años, Internet se usaba principalmente como herramienta para buscar información. En los últimos años, Internet se ha ido convirtiendo progresivamente en una gran red que lo abarca todo y que interconecta numerosos sistemas y dispositivos (Méndez, 2013). Esta nueva configuración de Internet es lo que se conoce como el "Internet de las Cosas".

El término Internet de las Cosas (Kopetz, 2011; Xia et al., 2012), es un concepto que nació en el Instituto de Tecnología de Massachusetts (MIT) a finales de los años noventa. Y trata sobre la capacidad de internet para operar con los datos generados por las cosas, en el que máquinas y personas están conectados y pueden comunicarse entre sí. Para esto se necesita que las cosas tengan conexión a Internet en cualquier momento y lugar. En un sentido más técnico, consiste en la integración de sensores y dispositivos en objetos cotidianos que quedan conectados a Internet a través de redes fijas e inalámbricas. (Baoyun, 2009; Gershenfeld et al., 2004)

Según los expertos, en menos de 10 años, las redes personales tendrán que hacer frente a miles de dispositivos conectados a ellas. Cualquier objeto, desde una computadora hasta un equipo electrodoméstico, deberá tener acceso en esta nueva red del futuro, que ya está dando sus primeros pasos (Gartner, 2017). El Internet del futuro se caracterizará por su ubicuidad y accesibilidad desde cualquier lugar, por cualquier persona o dispositivo conectado a ella, y en cualquier momento. (Tan

149 Vol. 4, núm. 2, abril, 2018, pp. 147-160

Marely Cruz Felipe, Jenmer M. Pinargote Ortega, Gabriel Demera Ureta, Dannyll Zambrano Zambrano 
Tecnologías de internet de las cosas en la obtención de información

and Wang, 2010) Este entorno que se presenta, puede tener numerosas ventajas, pero también se deberá tener en cuenta aspectos de gran importancia como la privacidad (García Salvatierra, 2012) y la seguridad, dos grandes retos de este nuevo modelo de comunicación. (Atzori et al., 2010)

Internet de las cosas puede definirse como una infraestructura de red global dinámica, con capacidades autoconfigurables, basadas en estándares y protocolos de comunicación donde los "objetos" físicos y virtuales llevan asociados una identidad, así como atributos físicos y personalidades virtuales, que se integran perfectamente en la red de la información.

El "Internet de las cosas" o "Internet de los objetos" (IoT, por sus siglas en inglés), según algunos expertos, plantean que no se trata de una revolución en las relaciones entre objetos y personas sino un paso en la evolución de la propia Internet sin embargo otros plantean que existe tal revolución en las relaciones entre los objetos y las personas, otros advierten que no es un incluso entre los objetos directamente, que se conectaran entre ellos y con la Red y ofrecerán datos en tiempo real. O, dicho de otro modo, se acerca la digitalización del mundo físico.

En el informe Horizon del NMC, se plantea como tecnologías que serán empleadas en la educación e investigación en América Latina entre los próximos años a internet de las cosas, lo cual estará dado en gran medida por la rápida proliferación de aplicaciones móviles en menos de un año. (Johnson et al., 2013)

\section{Materiales y métodos}

Para realizar la investigación se utilizó como método teórico, el analítico-sintético: a través del análisis se descompone un objeto o proceso en los diferentes elementos que lo integran para analizar, valorar y conocer sus particularidades lo cual fue aplicado en el análisis de las tecnologías de internet de las cosas existentes; a través de la síntesis se integran, valorándolos como un todo, teniendo en cuenta el enfoque de sistema, se aplica al sintetizar a partir del análisis anterior, las formas de obtención de información más conveniente de acuerdo a la aplicación de Internet de las cosas; se emplea como método empírico la observación visual.

150 Vol. 4, núm. 2, abril, 2018, pp. 147-160

Marely Cruz Felipe, Jenmer M. Pinargote Ortega, Gabriel Demera Ureta, Dannyll Zambrano Zambrano 


\section{Factores para el desarrollo de Internet de las cosas}

Décadas atrás, para realizar cualquier función propias de un ordenador se requería de dispositivos del tamaño de una habitación, en la actualidad un mayor número de operaciones son realizadas con facilidad por simples dispositivos electrónicos del tamaño de una gota de agua. El tamaño, el costo y el consumo de energía del hardware se han reducido drásticamente, por lo que ahora es posible fabricar dispositivos electrónicos diminutos a un costo muy reducido. Estos pequeños dispositivos, junto con la expansión de las redes de comunicación, permiten incorporar inteligencia y conexión a los objetos del mundo real y están transformando lo que era una red global de personas en una red global de todas las cosas conectadas entre sí.

Para la proliferación de internet de las cosas intervienen múltiples factores, como son (Everlet and Pastor, 2013; Taravilla Herrera, 2013):

- Popularización de placas de hardware libre y la potencia de cálculo de reducidos chips.

- Abaratamiento de sensores y etiquetas para objetos.

- Mejora de las comunicaciones y el internet inalámbrico de fácil acceso.

- La proliferación de nuevos dispositivos personales con inteligencia asociada.

- Plataformas IoT y abundancia de información compartida.

- Las posibilidades de conexión de la Ipv6, el 4G e Internet 0.

Con la unión de estos factores tenemos la posibilidad de incorporar Internet de las cosas en nuestras vidas.

\section{Tipos de objetos}

Internet de las cosas, es una idea que se basa en que exista un sistema de conectividad digital para cosas existentes, donde "cosas" se refiere a todo tipo de objetos cotidianos, e incluso a sus componentes. Los objetos o también llamados cosas se clasifican fundamentalmente en cuatro tipos: 
1. Objetos simples, "demasiado primitivos para ser conectados a Internet, pero que igual pueden participar en interacciones útiles"

2. Objetos sensitivos o comunicativos, "que contienen sensores que se pueden comunicar y dar información acerca de ellos mismos.

3. Objetos controlables, que cuentan con sensores "que pueden detectar, comunicar y recibir información y controlar.

4. Objetos considerados autónomos o inteligentes que cuentan con sensores y funciones sofisticadas.

De acuerdo al tipo de sensor presente en cada objeto determina las funciones de cada uno. Dentro de las funciones que realizan los sensores se pueden encontrar las de: ubicar y contextualizar objetos, estudiar su comportamiento y desencadenar acciones desde ellos. Estas tres acciones, estructuradas en la llamada "Pirámide de inteligencia de los objetos" (Taravilla Herrera, 2013), determinan qué grado de inteligencia poseen. En la figura 1 se relacionan los tipos de objetos con su nivel de inteligencia.

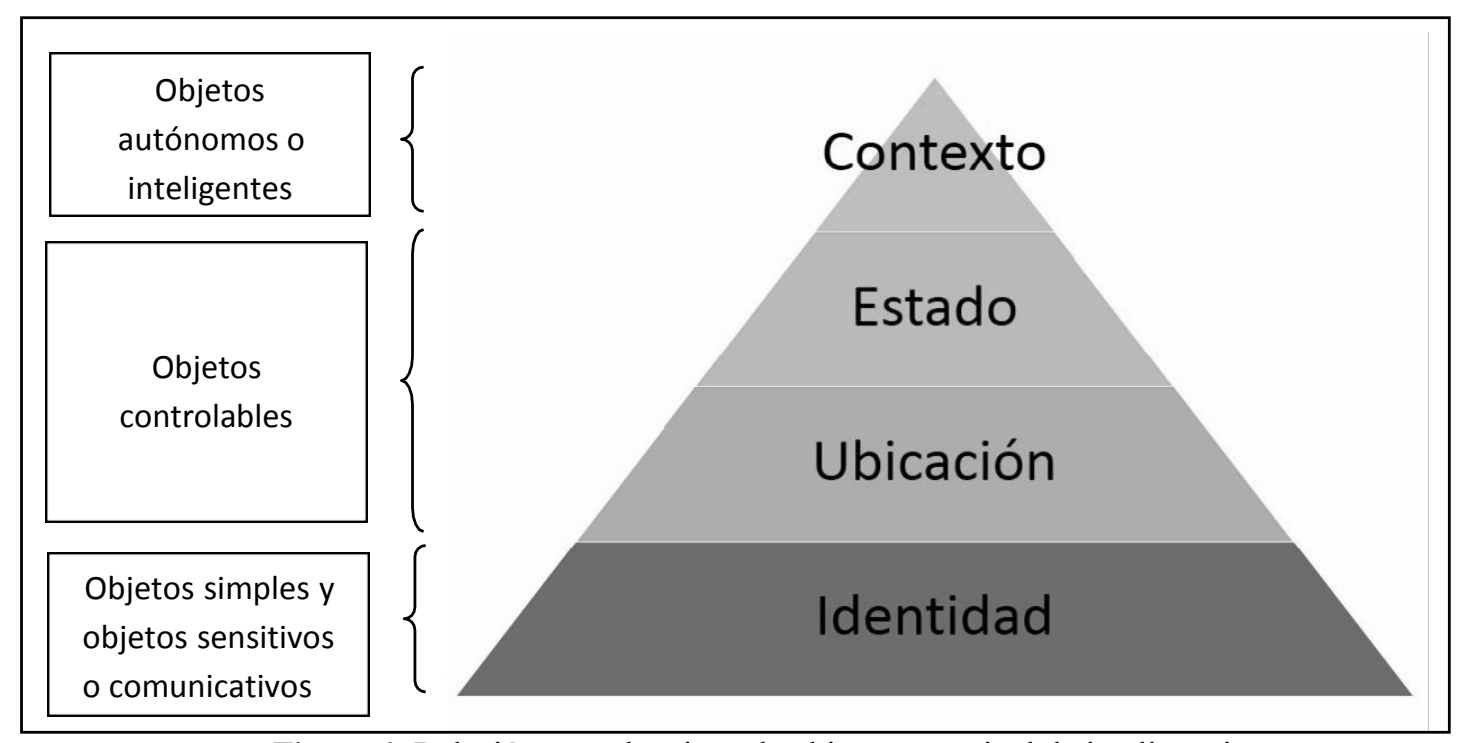

Figura 1: Relación entre los tipos de objetos y su nivel de inteligencia. 
Tecnologías de internet de las cosas en la obtención de información

- En el primer nivel, se ubican objetos simples y objetos sensitivos o comunicativos como por ejemplo una etiqueta RFID (Baoyun, 2009), donde cobran identidad única, es identificado con un número y una serie de propiedades primarias.

- El segundo nivel, está compuesto por objetos controlables y en este se informa de la trayectoria o posición del objeto a través de tecnologías como GPS.

- El tercer nivel también está compuesto por objetos controlables, se realiza la tarea de informar sobre el estado del objeto en un momento concreto y sus posibles cambios, a través de una conexión a la red dotada de tecnología $3 \mathrm{G}, 4 \mathrm{G}$ o afines.

- En el cuarto nivel, compuesto por objetos llamados autónomos o inteligentes, los objetos reciben información de su contexto con el objetivo de lograr interacción. En este los objetos pueden ser capaces de desencadenar acciones sobre su entorno. Este último paso es posible, al igual que el anterior, gracias a una conexión a la red junto a las otras características propias del objeto.

\section{Capas básicas de Internet de las cosas}

Para entender Internet de las cosas desde un punto de vista más técnico es necesario comprender las tres capas que lo componen, las mismas son (Bankinte, 2011):

1. Capa de Hardware: Más de medio siglo después de los pesados ordenadores que ocupaban salas enteras, los componentes son cada vez más pequeños, lo que permite desarrollar ordenadores más potentes y rápidos que sus predecesores. Esta capa física ocupa menos espacio, lo que facilita que se pueda conectar prácticamente cualquier cosa, desde cualquier sitio, en cualquier momento. Asistimos al fenómeno de la miniaturización.

2. La segunda capa es la infraestructura, o más bien sus limitaciones. Si miles de millones de nuevos dispositivos se conectan al IoT, ¿cómo lo soportará la tecnología actual? ¿Se va a convertir el espectro de Internet en el combustible del siglo XXI? Se hace necesario afrontar el problema del espectro como un recurso limitado.

153 Vol. 4, núm. 2, abril, 2018, pp. 147-160

Marely Cruz Felipe, Jenmer M. Pinargote Ortega, Gabriel Demera Ureta, Dannyll Zambrano Zambrano 
Tecnologías de internet de las cosas en la obtención de información

3. La tercera y última capa la forman las aplicaciones y los servicios que ponen en uso la gran cantidad de información creada a partir del IoT y donde se encuentra el mayor potencial de creación de valor. Estas aplicaciones conllevan la creación de nuevos modelos de negocio e iniciativas empresariales muy interesantes desde el punto de vista de la innovación.

Estas tres capas la miniaturización del hardware, las necesidades de infraestructura y el desarrollo de software innovador son fundamentales para entender la expansión del Internet de las Cosas en el terreno de las tecnologías de la información y la comunicación.

\section{Tecnologías empleadas}

Los sistemas basados en Internet de las cosas están compuestos de dispositivos, llamados objetos inteligentes, con prestaciones muy reducidas en cuanto a capacidad de memoria, batería, procesamiento y de comunicación. Además de esos objetos inteligentes, Internet de las cosas está compuesto de etiquetas y códigos de identificación que permiten la identificación única y a escala global de un determinado objeto o cosa. Para conformar estos sistemas, son varias las tecnologías disponibles que permiten el desarrollo de este tipo de objetos y recursos. De forma tal que se pueden dividir de acuerdo a sus funciones en dos tipos: Tecnologías para la identificación de objetos y tecnologías para el desarrollo y comunicación.

En la primera de estas tecnologías para la identificación de objetos o cosas, comenzó a extenderse por las técnicas impresas y dentro de ellas las tecnologías más extendidas históricamente son los códigos de barras. El código de barras permite llevar a cabo una identificación siempre de un recurso a nivel de identificador de tipo de producto y fabricante. Como una evolución al código de barras, han aparecido los códigos bidimensionales, los cuales ofrecen una mayor capacidad, permitiendo almacenar más información acerca del producto etiquetado y la creación de un identificador único a nivel de producto en concreto, así como su enlace con Internet a través de la inclusión de enlaces de Internet (URL - Universal Resource Locators).

Posterior a las técnicas impresas, un gran avance en las tecnologías para la identificación de objetos o cosas durante los últimos 20 años ha consistido en la tecnología para identificación por 
radiofrecuencia (RFID, Radio Frequency Identification). RFID ofrece múltiples ventajas a las tecnologías de identificación impresas, permiten que múltiples objetos o cosas puedan ser identificados simultáneamente, mediante ellas es posible identificar objetos que no se encuentran en la línea de visión, además ofrecen una mayor capacidad de almacenamiento de datos manteniendo un pequeño tamaño de la etiqueta. (Bayani, 2017)

Como una evolución de RFID (Yan et al., 2008) continuó la integración de RFID en los móviles para identificación de objetos dentro de un campo cercano (NFC, Near Field Communication). NFC ofrece las capacidades de RFID integradas en el móvil, y además incorpora funcionalidades extra como el establecimiento de comunicaciones entre dos dispositivos. (P2P - Peer-to-Peer)

Otras tecnologías y dispositivos existentes en el Internet actual son los dispositivos inteligentes un ejemplo de este tipo de dispositivos son los teléfonos inteligentes (smart phones), tabletas, computadoras portátiles, tecnologías industriales, electrodomésticos entre otros.

En segundo tipo de tecnologías compuesto por tecnologías para el desarrollo y comunicación en este caso de objetos inteligentes encontramos tecnologías como 6LoWPAN para redes de sensores (basadas en IEEE 802.15.4), Bluetooth Low Energy (IEEE 802.15.1) para redes de área personal, WiFi Low Power (IEEE 802.11) para redes de área local, y finalmente tecnologías celulares como Long Term Evolution Advanced (LTE-A) para comunicaciones máquina a máquina en redes de área extendida. (Jara Valera, 2013; Mercado et al., 2013)

Esta nueva concepción de extender Internet a todas las cosas es posible gracias a la nueva versión del protocolo de Internet: IPv6. IPv6 extiende el espacio de direccionamiento para ser capaz de albergar todas las cosas que están siendo conectadas a Internet. IPv6 ha sido diseñado para ofrecer comunicaciones seguras a los usuarios, así como movilidad a todos los dispositivos utilizados por ese usuario. De esa manera, el usuario puede estar conectado en cualquier momento y desde cualquier lugar de una forma segura. Esas características de IPv6 es lo que ha hecho posible pensar en conectar todos los objetos y crear un Internet de las cosas.

155 Vol. 4, núm. 2, abril, 2018, pp. 147-160

Marely Cruz Felipe, Jenmer M. Pinargote Ortega, Gabriel Demera Ureta, Dannyll Zambrano Zambrano 
El objetivo de Internet de las cosas es la integración y unificación de todas las comunicaciones, sistemas y dispositivos disponibles a nuestro alrededor. De esa manera los sistemas y dispositivos son capacidades de poder comunicarse con otros sistemas y dispositivos con el objetivo de poder ofrecer una nueva generación de servicios potenciados por las capacidades de la comunicación y computación ubicua. IPv6 ha sido considerada la tecnología más adecuada para el Internet de las cosas, ya que ofrece una alta escalabilidad, Internet ha sido probado de una forma intensiva durante los últimos años, está disponible en todas partes, es abierto y ofrece conectividad entre cualquier par de dispositivos conectados a Internet.

\section{Aplicaciones de Internet de las cosas}

El Internet de las Cosas se basa en conectar cualquier cosa, e impactará en todos los aspectos de nuestra sociedad, tales como educación, transporte, salud, monitoreo de signos vitales, deporte y entretenimiento (Arias, 2013). Tal es así que según muestran varios informes presentados diciembre 2013 en el foro IoT en Barcelona 'Internet de las cosas' hará que en 2020 cada persona tenga como media nueve dispositivos conectados a la Red, en lugar de los dos actuales. (Gómez, 2013)

Mediante el empleo tan simple de etiquetas RFID es posible seguir objetos, sensores para temperatura, calidad ambiental, humedad, detección de movimiento y conmutadores que pueden encender o apagar cosas como luces, calefactores, sistemas de riego, aires acondicionados, video cámaras, etc.)

Además, se destacan en el empleo de IoT objetos llamados inteligentes como los smart phones, smart Tvs, smart wach, smart home, entre otros con disimiles funciones y las smart city.

Smart phones: Es un teléfono móvil construido sobre una plataforma informática móvil, con una mayor capacidad de almacenar datos y realizar actividades semejantes a una minicomputadora y conectividad que un teléfono móvil convencional.

Smart TVs: Televisor inteligente que realiza múltiples funciones dentro de ellas acceder a internet. Se calcula que para el que para el año 2015 el 55\% de televisores sean televisiones inteligentes.

156 Vol. 4, núm. 2, abril, 2018, pp. 147-160

Marely Cruz Felipe, Jenmer M. Pinargote Ortega, Gabriel Demera Ureta, Dannyll Zambrano Zambrano 
Smart City: Es una ciudad que usa las tecnologías de la información y las comunicaciones para hacer que tanto su infraestructura crítica, como sus componentes y servicios públicos ofrecidos sean más interactivos, eficientes y los ciudadanos puedan ser más conscientes de ellos. Los servicios que brinda una smart city son: movilidad urbana, eficiencia energética y medioambiente, gestión de infraestructuras y edificios públicos, gobierno y ciudadanía, seguridad pública, salud, educación, capital humano y cultura, e-comercio. (Telefónica, 2011)

Según fue planteado en IoT Forum el IoT debe servir para que la conectividad y los datos nos ayuden a tomar decisiones, nos ahorren tiempo y nos hagan la vida más fácil. (Gómez, 2013)

\section{Conclusiones}

- Internet de las cosas muestra una forma nueva de acceder a la información a partir de objetos con posibilidades de ser censados y de establecer comunicación.

- Los objetos también llamados cosas se clasifican en objetos simples, sensitivos o comunicativos, controlables y los considerados autónomos o inteligentes esto su vez se ve reflejado en el nivel de inteligencia que poseen los objetos lo cual le permite definir la información que brindan como son definir su identidad, su ubicación el estado e interactuar sobre el contexto que lo rodea.

- $\quad$ Para hacer realidad Internet de las cosas, se requiere una evolución en la tecnología que soporta a Internet. Dentro de las tecnologías que se destacan se encuentran con posibilidades de brindar mayor cantidad de información las siguientes: para la identificación las RFID y dispositivos inteligentes que a la vez se puede establecer comunicación, y para la comunicación y desarrollo se pueden emplear tecnologías como 6LoWPAN, Bluetooth Low Energy, WiFi Low Power y Long Term Evolution Advanced.

- $\quad$ Las aplicaciones de internet de las cosas impactan en todos los sectores de la sociedad y es un fenómeno que tiende a introducirse de forma exponencial, sustentada sobre el desarrollo de las tecnologías y en especial las tecnologías inalámbricas, cada aplicación demanda de un conjunto de

157 Vol. 4, núm. 2, abril, 2018, pp. 147-160

Marely Cruz Felipe, Jenmer M. Pinargote Ortega, Gabriel Demera Ureta, Dannyll Zambrano Zambrano 
Tecnologías de internet de las cosas en la obtención de información

información que el uso de Internet de las cosas facilita y hace posible adaptar con facilidad de acuerdo a la aplicación que se desee desarrollar.

\section{Referencias Bibliográficas}

ARIAS, M. (2013). Integrando el mundo físico con el virtual: Internet de las Cosas. Ireland: National University of Ireland.

ATZORI, L., IERA, A., \& MORABITO, G. (2010). The internet of things: A survey. Computer networks, 54 (15): Pp. 2787-2805.

BANKINTER. (2011) El internet de las cosas. En un mundo conectado de objetos inteligentes [en línea]. Disponible en http://www.belt.es/expertos/imagenes/XV_FTF_El_internet_de_las_cosas.pdf

BAOYUN, W. (2009). Review on internet of things. Journal of Electronic Measurement and Instrument, 23(12): Pp. 1-7.

BAYANI, M.; SEGURA A., ALVARADO M. \& LOAIZA M. (2017) IoT-Based Library Automation and Monitoring system: Developing an Implementation framework of Implementation e-Ciencias de la Información, volume 8, number 1, Jan-Jun. [en línea]. Disponible en http://www.redalyc.org/jatsRepo/4768/476854589005/index.html

BECARES, B. (2014) Los hogares inteligentes, el Internet y de las Cosas y... ¿la privacidad? [en línea]. Disponible en http://www.channelbiz.es/2014/02/06/los-hogares-inteligentes-el-internet-y-delas-cosas-y-la-privacidad/?inf_by $=5$ aef3cfb671db8d6388b53c6

EVERLET, A. \& PASTOR, J. Introducción al Internet de las cosas. Construyendo un proyecto de IOT. España: Universidad Rey Juan Carlos. 2013.

GARCÍA, A. (2012) El Internet de las Cosas y los nuevos riesgos para la privacidad, E Telecomunicacion. 2012. 
Tecnologías de internet de las cosas en la obtención de información

GARTNER, INC, (2017). Gartner says 8.4 billion connected "things" will be in use in 2017, up 31 percent from 2016 [en línea] . Disponible en http://www.gartner.com/ newsroom/id/3598917

GERSHENFELD, N., KRIKORIAN, R., COHEN, D. (2004). The Internet of things. Scientific American, 291 (4): Pp. 76-81.

GÓMEZ, C. (2013). Tendencias 21. Tendencias de las Telecomunicaciones. Rev. Electrónica Cienc. Tecnol. Soc. Cult.

JARA, A. (2013) Propuesta de una arquitectura de red basada en internet de las cosas para la integración ubicua de entornos clínicos con soporte escalable a la seguridad y la movilidad, Universidad de Murcia. Proyecto de Investigación. [en línea] Disponible en https://digitum.um.es/xmlui/handle/10201/36978

JOHNSON, L., TORRES, S., ANTÓN, J., BECKER, S., DESENNE, P., NÓ, J., ET AL. NMC (2013). Perspectivas Tecnológicas: Educación Superior en América Latina 2013-2018. Un Análisis Reg. Inf. Horiz. NMC. 2013.

KOPETZ, H. (2011). Internet of things. en: Real-Time Systems. Spring. Pp. 307-323.

MÉNDEZ, I. G. (2013). Hacia el "todo conectado": analizamos los nichos de negocio que se abren con el internet de las cosas Emprendedores: las claves de la economía y el éxito profesional, (191): Pp. 92-96.

MERCADO, G., BORGO, R., GONZALEZ, F., TAFFERNABERRY, J., DIEDRICHS, A., AGUIRRE, M., ET AL. (2013). Aplicaciones de Internet de las Cosas SIPIA6-Red de Sensores Inalámbricos con IPv6. XV Workshop de Investigadores en Ciencias de la Computación. Pp. 43-47.

Pp. 1101-1102.

TAN, L. \& WANG, N. (2010). Future internet: The internet of things. Advanced Computer Theory and Engineering (ICACTE), 3rd International Conference on, IEEE, 
TARAVILLA, J. (2013) El futuro de la red: Internet de las cosas. Creat.-Commons Contrib. [en línea]. Disponible en https://horizontesdecompromiso.files.wordpress.com/2013/01/aqui3.pdf

TELEFÓNICA. (2011) Smart Cities: un primer paso hacia la Internet de las Cosas. España. Fundación Telefónica.

XIA, F., YANG, L. T., WANG L., \& VINEL, A. 2012. Internet of things. International Journal of Communication Systems, 25 (9)

YAN, L.; Y. ZHANG, L. YANG, \& H. NING. (2008) The Internet of Things: from RFID to the nextgeneration pervasive networked systems. CRC Press. 